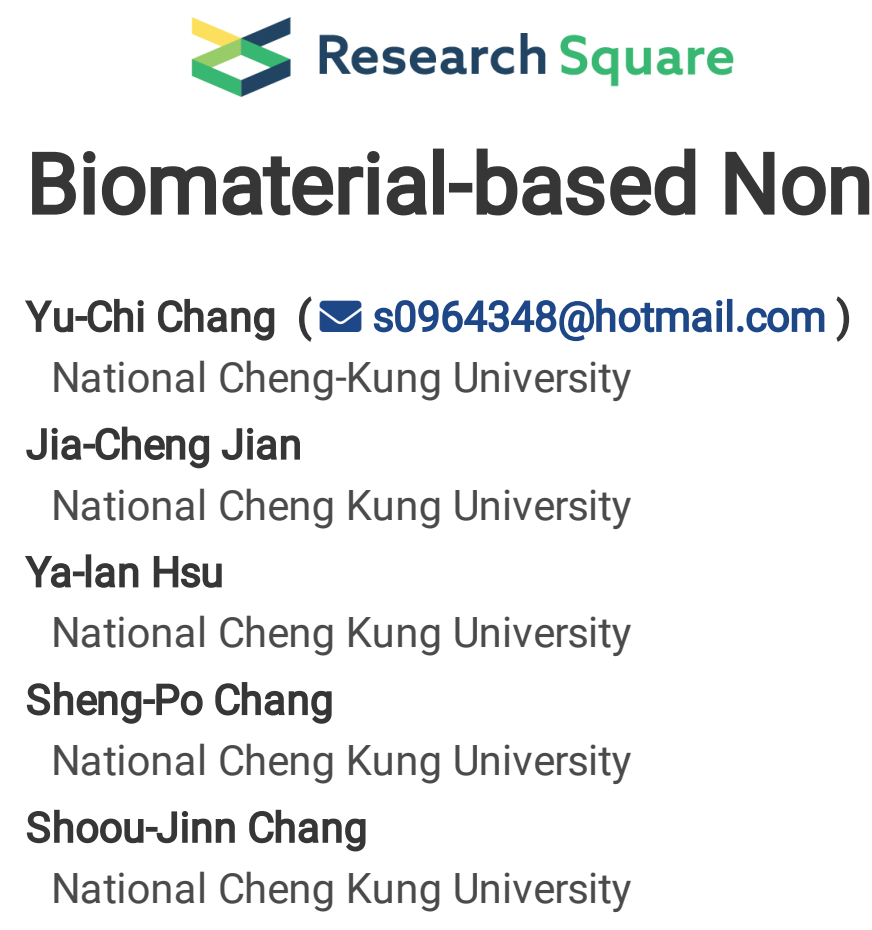

\author{
Yu-Chi Chang ( $\sim$ s0964348@hotmail.com ) \\ National Cheng-Kung University \\ Jia-Cheng Jian \\ National Cheng Kung University \\ Ya-lan Hsu \\ National Cheng Kung University \\ Sheng-Po Chang \\ National Cheng Kung University \\ Shoou-Jinn Chang \\ National Cheng Kung University
}

\title{
Biomaterial-based Nonvolatile Photonic Memristor
}

\section{Article}

Keywords: photonic memristor, nonvolatile data, photonic and neuromorphic computing

Posted Date: August 17th, 2021

DOI: https://doi.org/10.21203/rs.3.rs-798594/v1

License: (c) (1) This work is licensed under a Creative Commons Attribution 4.0 International License.

Read Full License 


\section{Abstract}

A photonic memristor is a component used in photonic and neuromorphic computing that can complete the high-speed programming of nonvolatile data through light illumination`. To date, photonic memristors have been fabricated using several methods. Applied principles include the photovoltaic (PV) effectmediated Schottky barrier, PV effect-induced formation/annihilation of conductive filaments, photogating effect and photoinduced chemical reaction/conformation change5. However, currently proposed solutions are half sets only (i.e. either light writing or light erasing only), photoinduced electrical programming or photovoltaically modulated6-9. Moreover, these devices frequently require the use of special materials or complex device structures and circuitries5. Here, we used a mixture of apple pectin (AP) and nickel oxide (NiO) nanoparticles (NPs) as the resistive switching layer and fabricated a simple metal/insulator/metal sandwich structure. The surface defects between the interface of AP and NiO NPs can capture ultraviolet-excited electrons and convert the resistance state into low-resistance state (LRS). By expelling the electrons in the traps through green light, LRS can be transformed back into highresistance state. The memory can be programmed purely through light and is compatible with electrical operation. This discovery provides a reliable method for fabricating photonic memristors that can be adopted in photonic and neuromorphic computing applications.

\section{Introduction}

In accordance with previous research, we determine that an apple pectin (AP) film is a biopolymer-based material that can be applied to nonvolatile resistive random-access memory (ReRAM) ${ }^{10}$. Studies have shown that the resistive switching (RS) behavior of AP is largely completed by the formation and rupture of conductive filaments (CFs) ${ }^{10}$. ReRAM made of biopolymer-based materials cannot be operated via light illumination. However, such hydrophilic material is highly suitable as a matrix for metal oxide nanoparticles (NPs). By combining with materials that are light-responsive to specific wavelengths, such as nickel oxide (NiO) NPs, an AP film can acquire the characteristics of mixed materials. The energy bandgap of $\mathrm{NiO}$ is approximately $3.3-4.0 \mathrm{eV}$, and it exhibits absorption characteristics for ultraviolet (UV) light and can be excited by light to generate photoelectrons ${ }^{11,12}$. Moreover, the surface defects of $\mathrm{NiO}$ NPs can be created via UV light illumination ${ }^{13}, 14$; such defects play an important role in this structured photonic memristor. By controlling the thickness of the mixed layer and the density of NiO NPs in the film, light-induced RS behaviour of the device can be achieved by filling and expelling carriers from these defects. Here, we use $350 \mathrm{~nm}$ UV light to excite $\mathrm{NiO}$ to generate photoelectrons that can easily fill the trap surrounding the surface of NPs. In addition, the trapped electrons can also be excited by a special wavelength of light and then expelled from the traps. On the basis of the trap-detrap transmission mechanism, the use of light illumination with different wavelengths can cause the device to demonstrate light-writing and light-erasing behavior, achieving the light operation of a photonic memristor. The preparation method for an AP film combined with NiO NPs is implemented through a simple solution process. Consequently, the ultrathin thickness of the AP film can only hold the size of NiO NPs (Figs. 1a and 1b). A room-temperature photoluminescence (PL) analysis (Fig. 1c) determines that the broad PL 
spectra consist of components centered at approximately 2.17, 2.30, 2.86 and $2.99 \mathrm{eV}$, as indicated by red arrows. In general, the components arise from the surface defects of NiO NPs ${ }^{15}, 16$. These surface defects effectively trap photoexcited electrons and subsequently hinder recombination from holes in this device structure.

To understand the photo-response of a photonic memristor device, the device was firstly irradiated by the continuous modulation of light with a wavelength ranging from $650 \mathrm{~nm}$ to $300 \mathrm{~nm}$. The reading voltage $\left(\mathrm{V}_{\text {read }}\right)$ was set to $-0.1 \mathrm{~V}$ to measure the current of the device at a sampling rate of $1 \mathrm{~s}$ (Fig. 2a). The results clearly showed that the current level can be divided into three states: P-low-resistance state (LRS)$f, P$-LRS-h and high-resistance state (HRS). The resistive state of the device changes into HRS when the wavelength of light illumination is $500 \mathrm{~nm}$. HRS represents the state in which $500 \mathrm{~nm}$ light can erase the memory device from LRS; to activate the light-erasing behaviour, the current level is switched from approximately $10-4 \mathrm{~A}$ to $10-7 \mathrm{~A}$. Meanwhile, the light-writing behaviour can be observed in the wavelength range between $650-550 \mathrm{~nm}$ and $<400 \mathrm{~nm}$. Regardless of whether for light-writing or lighterasing operation, the current switch will have an intermediate state (i.e. P-LRS-h) because of the incomplete structure of the current transmission path provided by the trap-detrap mechanism. The device has been confirmed to exhibit multilevel data storage capability, but further research on this feature has not been conducted.

Figure $2 \mathrm{~b}$ shows the $\mathrm{I}-\mathrm{V}$ characteristics of the device when the device was under dark condition and after UV light illumination. $A \pm 5 \mathrm{~V} \mathrm{I-V}$ double sweeping is performed under dark condition, and the set and reset voltages of the device are obtained as $+2 \mathrm{~V}$ and $-2 \mathrm{~V}$, respectively. The on/off current ratio is approximately $1.34 \times 10^{4}$ at $-0.1 \mathrm{~V}$. Notably, the device does not apply any voltage under UV light illumination to avoid photoinduced electrical programming. After UV light illumination for $60 \mathrm{~s}$ and $\mathrm{I}-\mathrm{V}$ single sweeping is performed from 0 to $-5 \mathrm{~V}$, the device is in LRS and its erasing process is performed after $-0.8 \mathrm{~V}$ until $-5.0 \mathrm{~V}$. Under dark condition, the device exhibits sharp transitions in the reset process. The erasing process after illumination presents typical trap-detrap negative differential resistance characteristics $^{17,18}$, and current gradually decreases after $-0.8 \mathrm{~V}$ as voltage increases. In accordance with our previous study ${ }^{10}$, the RS behaviour of an AP memory device is related to the formation and rupture of filament paths. To understand the current transfer mechanism clearly, linear log-log $I-V$ fitting is performed (Figs. 2c-2e).

In DARK-HRS (Fig. 2C), a segmented linear relation was observed in the plot of $\log (\mathrm{I})$ versus $\log (\mathrm{V})$ for a voltage sweeping performed from 0 to $1.45 \mathrm{~V}$, with slopes of 1.20, 2.14 and 12.07, suggesting that the space-charge-limited current (SCLC) dominates the carrier transport process ${ }^{19-21}$. In DARK-LRS (Fig. 2d), the plot from 0 to $2 \mathrm{~V}$ was fitted to a straight line. Such linear characteristic suggests that the conduction mechanism is probably due to ohmic conduction ${ }^{21}$. This result indicates that the transmission path created by voltage is in the form of CFs. In the AFTER UV-LRS curves (Fig. 2e), a similar relation was also observed in the plot for voltage sweeping performed from 0 to $1.45 \mathrm{~V}$, with slopes of 1.01, 1.49 and 3.97, suggesting the SCLC model. The fitting results of DARK-HRS and AFTER UV-LRS are both SCLC models, 
and this result corresponds to the current transmission path caused by the trap-detrap mechanism. Upon electrical programming, the RS behaviour of an AP memory device is related to the formation and rupture of filament paths. However, the phenomenon of current transmission conforms to the trap-detrap mechanism during light writing. This difference in results can prove that light irradiation creates a current transmission path that differs from the filament type during light writing.

To investigate the conductivity distribution and effect of light illumination on RS properties, conductive atomic force microscopy (CAFM) measurements were simultaneously performed on ITO/NiO NPs-AP surface under dark condition and after $350 \mathrm{~nm}$ UV light illumination. A circular pattern with a low current value was observed, and the position of the low current pattern should be the distribution position of accumulated NiO NPs (Fig. 3a). After light illumination (Fig. 3b), the state without peak current was maintained and overall current value was increased. The current increase rate at the distribution position of the accumulated NiO NPs is also higher than that in other positions (Fig. 3c). This result indicates that the current transmission path generated after light illumination must be distributed in the position of the accumulated NiO NPs. Moreover, it is not a filament-type current transmission path. This finding is consistent with the results of the $\mathrm{I}-\mathrm{V}$ curve fitting.

In accordance with the analysis results, the major current conduction mechanism of an AP film is filament theory, and the current conduction path generated by light illumination mostly follows the trapdetrap mechanism. The current conduction paths are summarized as follows: Type I (filament only), Type II (trap-assisted tunneling and trap-detrap domain) and Type III (hybrid path, as shown in Figs. 4a and 4b). Type I transmission paths are distributed in locations that are largely composed of APs without NiO NPs. This transmission mechanism is the same as that of the AP film, and it requires the application of voltage to drive the formation of CFs. Type II is mostly distributed in the location of NiO NPs, and its transport mechanism is dominated by defects on the surface of NiO NPs. Figure 4c plots the surface defect and photoelectron behavior of NiO NPs. When the memory device is irradiated by UV light with a wavelength of $350 \mathrm{~nm}$, NiO NP absorbs the light and generates excitons. Amongst them, the photoelectron transitions from the valence band (VB) of $\mathrm{NiO}$ to the conduction band (CB) after being excited. The photoelectron immediately drifts towards the interface between AP and NiO NPs due to the bending of the NiO energy band and gets trapped in the surface defect of NiO NPs. Such behaviour leads to the light-writing phenomenon of the memory device. As illustrated in Fig. 4d, when the device is irradiated with green light with a wavelength of $500 \mathrm{~nm}$, trapped electrons are excited back to CB and then back to VB to recombine with holes. Evidently, the light-erasing phenomenon of the device is achieved after reverse light excitation and recombination. Type III combines the transmission paths of Types I and II, which primarily occur at the interface of the AP material and NiO NPs. As indicated in the literature, when the filament is described as Type I, the rupture part of the filament will remain. The rupture part of CFs mostly occurs close to the Al top electrode, whilst CFs close to the ITO bottom electrode remain. Therefore, the current is likely to be transmitted through the partial transmission channels of Types I and II. From the preceding mechanism, this device can perform reliable and stable light programming via light illumination with different wavelengths. 
To confirm the repeatability and stability of light programming further, the endurance and retention characteristics of the memory device were tested. Figures $5 \mathrm{a}$ and $5 \mathrm{~b}$ respectively show the RS behavior of light writing with electrical erasing and light writing with light erasing. To avoid light-assisted programming, $\mathrm{V}_{\text {read }}$ was not applied during illumination. At other times, $\mathrm{V}_{\text {read }}$ was set to $-0.1 \mathrm{~V}$ with a sampling rate of once per second, and $V_{\text {erasing }}$ was set to $-3.0 \mathrm{~V}$. Illumination time was set to $60 \mathrm{~s}$, and light wavelengths of $350 \mathrm{~nm}$ and $500 \mathrm{~nm}$ correspond to UV light writing and green light erasing, respectively. In Figs. $5 a$ and $5 b$, the average on/off current ratio can reach more than $5 \times 10^{2}$, and repeatable RS behaviour can be obtained. Although the operations of light writing with electrical erasing and light writing with light erasing presented only seven and nine RS cycles, respectively, the device can still be determined as controllable, reversible and reproducible via light operation.

Figure $5 c$ shows the retention characteristics of LRS after light writing (P-LRS-f), LRS after electrical writing and HRS. Evidently, resistance in the P-LRS-f, LRS and HRS states was demonstrated to remain for $10^{4} \mathrm{~s}$, and the on - off ratio was $>10^{3}$. The excellent retention properties of the memory device demonstrate that NiO NPs in AP is a feasible composition for photonic memristors.

\section{Conclusions}

In summary, we successfully fabricated a photonic memristor based on NiO NPs embedded into AP through a simple solution process technique. The results of using electrical writing and UV light writing exhibited different current transmission mechanisms, clearly confirming the uniqueness of the lightwriting behavior. In addition, light erasing can be achieved during green light irradiation with a wavelength of $500 \mathrm{~nm}$. These phenomena were confirmed to be related to the filling and expelling of photoelectrons on the surface defects of NiO NPs. Therefore, a photonic memristor can perform nonvolatile light-writing and light-erasing operations without voltage assistance. The realization of a complete photonic memristor has achieved progress in the future applications of photonic and neuromorphic computing.

\section{Methods}

Materials. Apple pectin was supplied by Sigma-Aldrich. Nickel oxide dispersion was supplied by US Research Nanomaterials.

Sample preparation. The apple pectin solution and $\mathrm{NiO}$ nanoparticle dispersions were used without further purification. ITO coated flexible glass substrates were cut to $2.0 \mathrm{~cm} \times 1.5 \mathrm{~cm}$ and cleaned with acetone, methanol, and de-ionized water in an ultrasonic bath. The pectin mixed with $\mathrm{NiO}$ nanoparticle solution spun on the cleaned ITO/Glass substrates and baked at $60^{\circ} \mathrm{C}$ for 15 minutes. Finally, an Al metal was deposited by RF sputtering for electrical measurements.

Instrumentation. The electrical properties were characterized by an Keithley 2636B system source meter. Current sensing atomic force microscopy (CSAFM) (Dimension ICON with Nano Scope V controller, Bruker, USA)) was used to characterize the current and surface morphology of the obtained ITO/NiO NPs- 
AP/Al memory samples. Transmission electron microscope (TEM) analysis was carried out using JEM2100F Electron Microscope. Photoluminescence (PL) (Jobin Yvon/Labram HR)was used to identify the electronic bandgap.

\section{Declarations}

\section{Competing interests}

The authors declare no competing interests.

\section{Acknowledgments}

This research is sponsored by the Ministry of Science and Technology of Taiwan under Grant no. MOST 109-2636-E-006-023.

\section{References}

1. Zhang, K., Meng, D., Bai, F., Zhai, J., and Wang, Z. L. Photon-Memristive System for Logic Calculation and Nonvolatile Photonic Storage. Advanced Functional Materials 30, 2002945 (2020).

2. Kumar, M., Abbas, S., \& Kim, J. All-oxide-based highly transparent photonic synapse for neuromorphic computing. ACS applied materials \& interfaces 10, 34370-34376 (2018).

3. Hu, L., Yang, J., Wang, J., Cheng, P., Chua, L. O., and Zhuge, F. All-optically controlled memristor for optoelectronic neuromorphic computing. Advanced Functional Materials 31, 2005582 (2021).

4. Emboras, A. et al. Opto-electronic memristors: Prospects and challenges in neuromorphic computing. Applied Physics Letters 117, 230502 (2020).

5. Mao, J. Y., Zhou, L., Zhu, X., Zhou, Y., and Han, S. T. Photonic memristor for future computing: a perspective. Advanced Optical Materials 7, 1900766 (2019).

6. Tan, H. et al. An optoelectronic resistive switching memory with integrated demodulating and arithmetic functions. Advanced Materials 27, 2797-2803 (2015).

7. Tran, M. D.et al. Two-terminal multibit optical memory via van der Waals heterostructure. Advanced Materials 31, 1807075 (2019).

8. Lv, Z. et al. Phototunable Biomemory Based on Light-Mediated Charge Trap. Advanced Science 5, 1800714 (2018).

9. Bera, A., Peng, H., Lourembam, J., Shen, Y., Sun, X. W., and Wu, T. A versatile light-switchable nanorod memory: wurtzite ZnO on perovskite SrTiO3. Advanced Functional Materials 23, 4977-4984 (2013).

10. Chang, Y. C., Jian, J. C., Chuang, M. Y., Hsu, Y. L., Huang, W. Y., and Young, S. J. Metal and Carbon Filaments in Biomemory Devices through Controlling the Al/Apple Pectin Interface. ACS Applied Electronic Materials 2, 2798-2805 (2020). 
11. Fu, Y. et al. All-solid-state Z-scheme system of NiO/CDs/BiVO4 for visible light-driven efficient overall water splitting. Chemical Engineering Journal 358, 134-142 (2019).

12. Hu, C. C., and Teng, $\mathrm{H}$. Structural features of p-type semiconducting $\mathrm{NiO}$ as a co-catalyst for photocatalytic water splitting. Journal of Catalysis 272, 1-8 (2010).

13. Rashad, M., Darwish, A. A. A., Qashou, S. I., and Abd El-Rahman, K. F. Influence of ultraviolet irradiation on physical properties of nano-NiO films for optical applications. Applied Physics A 126, 1-9 (2020).

14. Wang, A. et al. Vacancy defect modulation in hot-casted NiOx film for efficient inverted planar perovskite solar cells. Journal of Energy Chemistry 48, 426-434 (2020).

15. Deshpande, M. P., Patel, K. N., Gujarati, V. P., Patel, K., \& Chaki, S. H. Structural, thermal and optical properties of nickel oxide $(\mathrm{NiO})$ nanoparticles synthesized by chemical precipitation method. Advanced Materials Research 1141, 65-71 (2016).

16. Musevi, S. J., Aslani, A., Motahari, H., and Salimi, H. Offer a novel method for size appraise of NiO nanoparticles by PL analysis: Synthesis by sonochemical method. Journal of Saudi Chemical Society 20, 245-252 (2016).

17. $\mathrm{Ma}, \mathrm{H}$. et al. Interface state-induced negative differential resistance observed in hybrid perovskite resistive switching memory. ACS applied materials \& interfaces 10, 21755-21763 (2018).

18. Zhang, T. et al. Negative differential resistance, memory, and reconfigurable logic functions based on monolayer devices derived from gold nanoparticles functionalized with electropolymerizable TEDOT units. The Journal of Physical Chemistry C121, 10131-10139 (2017).

19. Liu, Y., Li, F., Chen, Z., Guo, T., Wu, C., and Kim, T. W. Resistive switching memory based on organic/inorganic hybrid perovskite materials. Vacuum 130, 109-112 (2016).

20. Harada, T. et al. Trap-controlled space-charge-limited current mechanism in resistance switching at Al/Pr 0.7 Ca 0.3 Mn 03 interface. Applied Physics Letters 92, 222113 (2008).

21. Jin, Z., Liu, G., \& Wang, J. Organic nonvolatile resistive memory devices based on thermally deposited Au nanoparticle. Aip Advances 3, 052113 (2013).

\section{Figures}



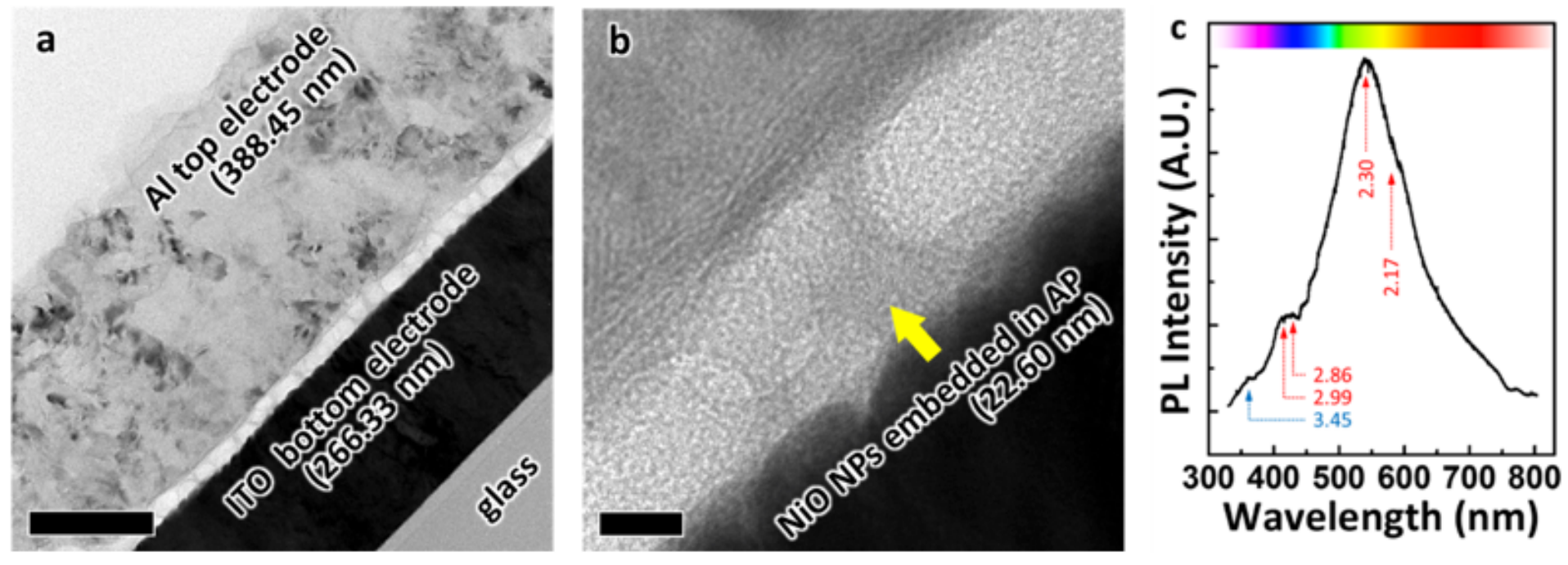

Figure 1

Basic properties of NiO NPs embedded into AP thin film. a, Low-magnification cross-sectional TEM image of the ITO/NiO NPs-AP/Al structure. Scale bar: $200 \mathrm{~nm}$. b, High-magnification cross-sectional TEM image of NiO NPs embedded into AP thin film. Scale bar: $10 \mathrm{~nm}$. c, Room-temperature PL spectra of NiO NPs-AP thin film. 

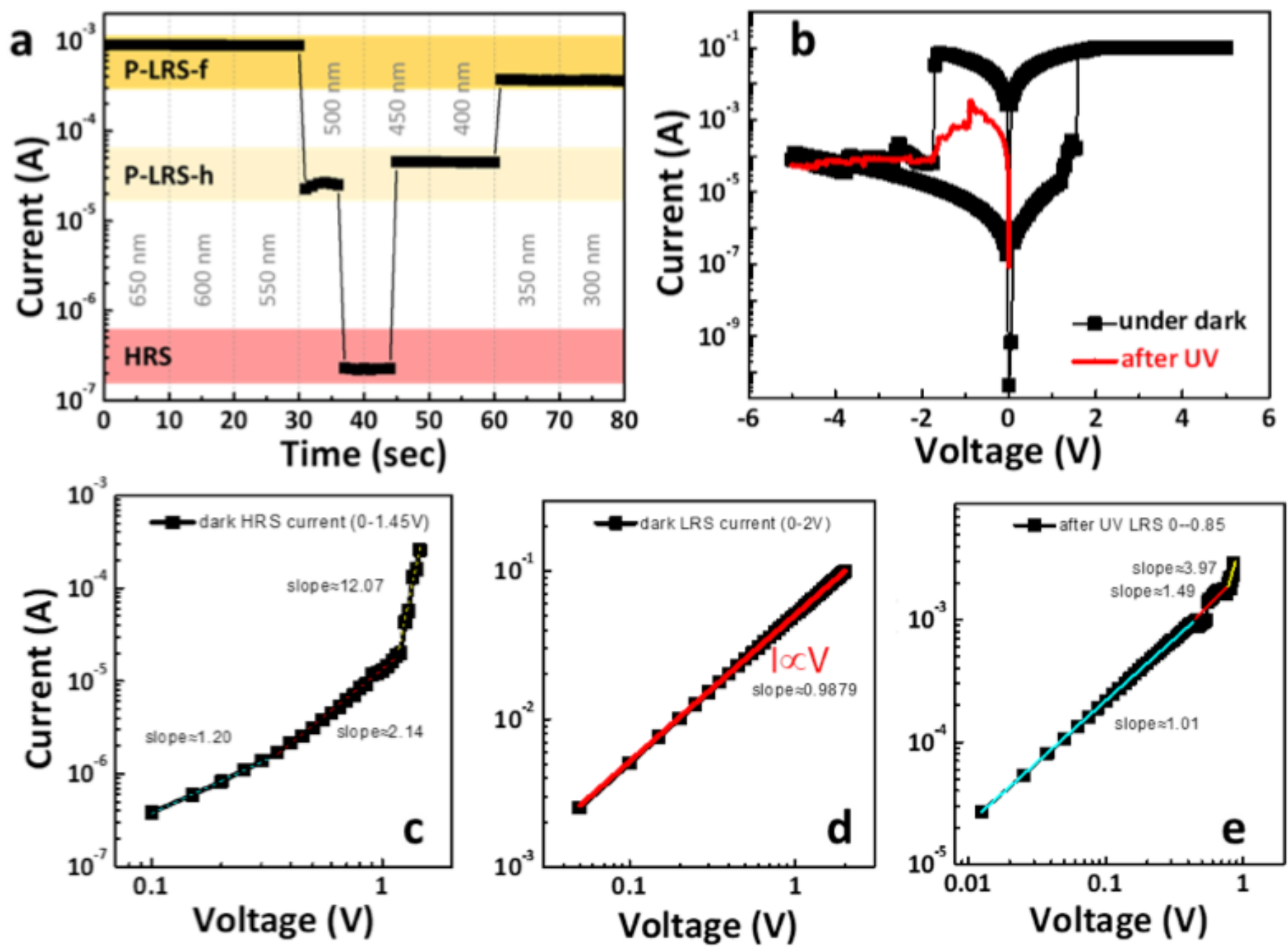

Figure 2

Photo induced RS behavior and I-V characteristics a, RS behavior of the ITO/NiO NPs-AP/AI memory device irradiated by a continuous modulation of light from $650 \mathrm{~nm}$ to $300 \mathrm{~nm}$ wavelengths. b, I-V characteristics of the ITO/NiO NPs-AP/Al memory device when the device is under dark condition (black) and after $350 \mathrm{~nm}$ UV light illumination (red). c, Log-log scale fitting of the HRS I-V curve from 0 to 1.45 $\mathrm{V}$ (DARK-HRS). d, Log-log scale fitting of the LRS I-V curve from 0 to $2 \mathrm{~V}$ (DARK-LRS). e, Log-log scale fitting of the P-LRS-f I-V curve from 0 to $-0.85 \mathrm{~V}$ (AFTER UV-LRS).
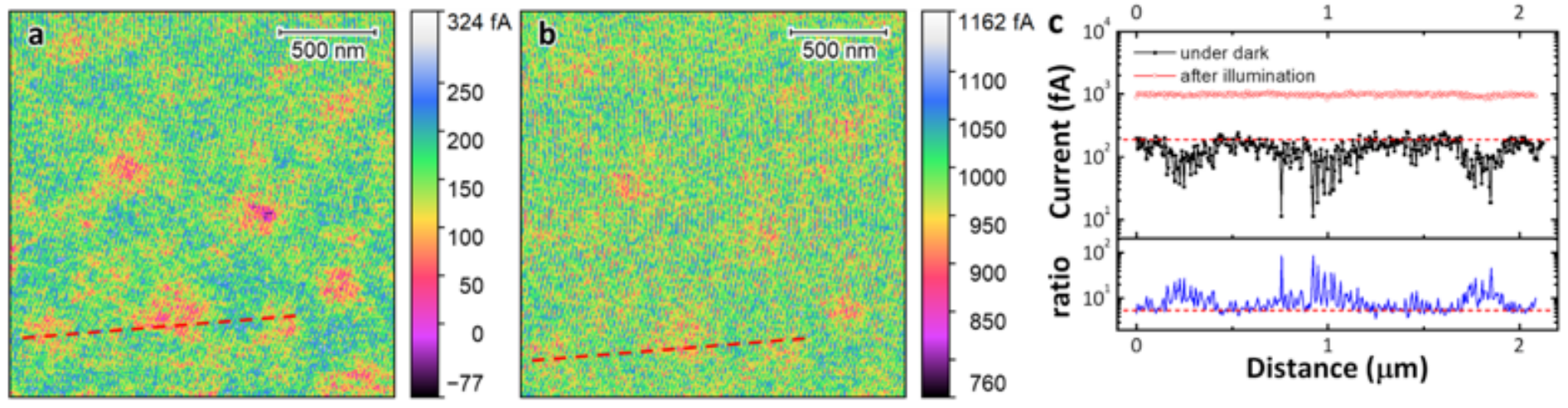


\section{Figure 3}

CAFM results a, Tunnelling atomic force microscopy (TUNA) current image of ITO/NiO NPs-AP under dark condition with an applied voltage of $2 \mathrm{~V}$. b. TUNA current image of ITO/NiO NPs-AP after light illumination with an applied voltage of $2 \mathrm{~V}$. c, Current distributions from the dashed line of Images a and b and the ratio of the currents.
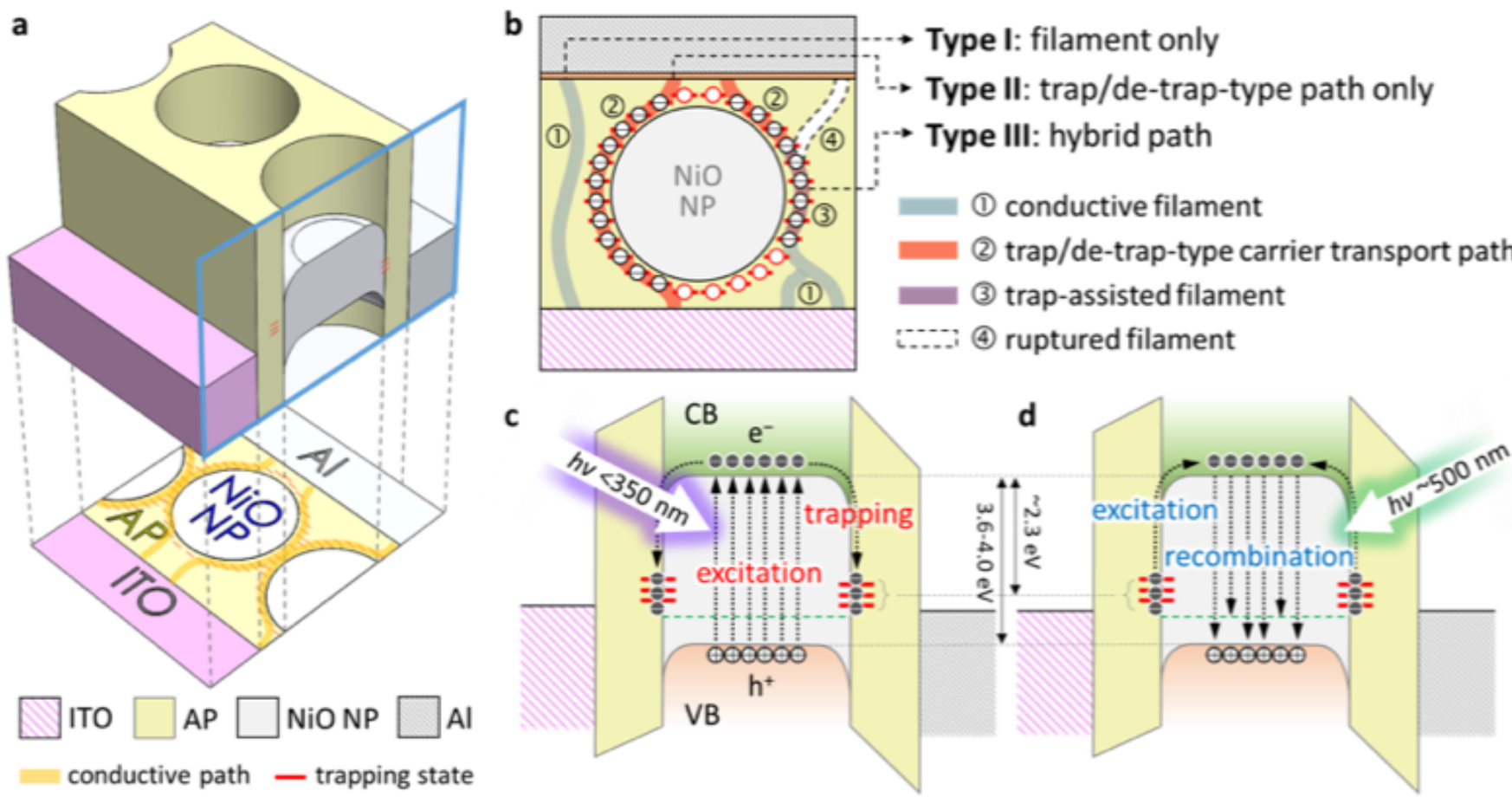


\section{Figure 5}

Repeatability and stability of the device a, Endurance performance of the memory device. Application of $350 \mathrm{~nm}$ UV light to switch the memory state to $\mathrm{ON}$ and $-3 \mathrm{~V}$ to switch it back to OFF. Vread is $-0.1 \mathrm{~V}$. b, Application of $350 \mathrm{~nm}$ UV light to switch the memory state to $\mathrm{ON}$ and $500 \mathrm{~nm}$ green light to erase the device. Vread is $-0.1 \mathrm{~V}$. c, Retention performance of the memory device at $1 \times 104$ s under a reading voltage of $-0.1 \mathrm{~V}$. 